\title{
Circulation in Rapid Hip and Knee Bone Destruction
}

\author{
Fred Ritchie Trew Nelson, MD ${ }^{1}$ \\ ${ }^{1}$ Department of Orthopaedics, Henry Ford Hospital, Detroit, \\ Michigan, United States
}

J Hip Surg 2017;1:55-60.
Address for correspondence Fred Ritchie Trew Nelson, MD,
Department of Orthopaedics, Henry Ford Hospital, 2799 West Grand
Boulevard, Detroit, Ml 48202, United States (e-mail: fnelson1@hfhs.org).

\begin{abstract}
Keywords

- subchondral insufficiency fracture

- hip

- knee

- rapidly developing osteoarthritis

- vascularity

- cytokine

In the hip and knee, the term subchondral insufficiency fracture has been used to describe a focal bone response associated with sudden onset of joint pain, older age, focal osteoporosis, and progression to joint replacement in some cases. Rapidly developing osteoarthritis of the hip is also associated with sudden onset of severe pain, and it usually progresses to larger areas of bone resorption with progression to joint replacement. The literature relative to these three conditions was reviewed focusing on patient demographics, imaging, osteoporosis, associated comorbidities, cytokine changes, similar vascular disorders in other joints, and attempts at nonsurgical treatment. These conditions predominate in, but are not restricted to, individuals who are older than 50 , have physical joint abnormalities, are not systemically osteoporotic, and have had no apparent trauma. The biochemical and genetic mechanisms for hip and knee insufficiency fractures is not clear. Although there are reports of hip subchondral insufficiency fractures associated with rapidly developing osteoarthritis of the hip, it is not clear whether all cases are preceded with insufficiency fractures. There is insufficient information on the fracture healing mechanism of insufficiency fractures, and the relationship to vascularity and biochemical imbalance is poorly understood. It is concluded that there are large gaps in the knowledge of biochemical and other mechanisms that might link these conditions. This knowledge may influence nonsurgical treatment strategies.
\end{abstract}

\section{Purpose}

The purpose of this review was to define subchondral insufficiency fracture of the hip (SIFH) and subchondral insufficiency fracture of the knee (SIFK), describing similarities and disparities as it may relate to circulatory dynamics. SIFH may lead to rapidly destructive osteoarthritis (RDO) of the hip. SIFH is likely different from the somewhat similar, but not necessarily related, focal sequence of events in the knee with SIFK. Typically, both start as a "subchondral insufficiency fracture" (SIF) and the clinical course is not entirely predictable. Whereas an insufficiency fracture of the hip SIFH may lead to RDO with femoral head and acetabular bone loss, SIFK will affect only a portion of the condyle or tibial plateau of the knee. In addition, there are instances of RDO that are not initially identified as SIFH. ${ }^{1,2}$ The purpose of this review was to explore clinical comparisons of SIF in the hip and knee outlining the relationship to bone edema seen on magnetic resonance imaging (MRI) and biochemical and histologic changes. Within this context, cytokine signaling, possible vascular involvement, current treatments, and possible future therapies are discussed.

\section{Subchondral Insufficiency Fracture of the Hip and Rapidly Destructive Osteoarthritis of the Hip}

Most major joint replacement surgeons have encountered what they initially thought was hip osteonecrosis (ON) only to discover a subsequent pattern of femoral head bone
Guest Editors: Lynne C. Jones, PhD and Michael A. Mont, MD
Copyright $\odot 2017$ by Thieme Medical Publishers, Inc., 333 Seventh Avenue, New York, NY 10001, USA. Tel: +1(212) 584-4662.
DOI http://dx.doi.org/ 10.1055/s-0037-1602181. ISSN 2472-8446. 


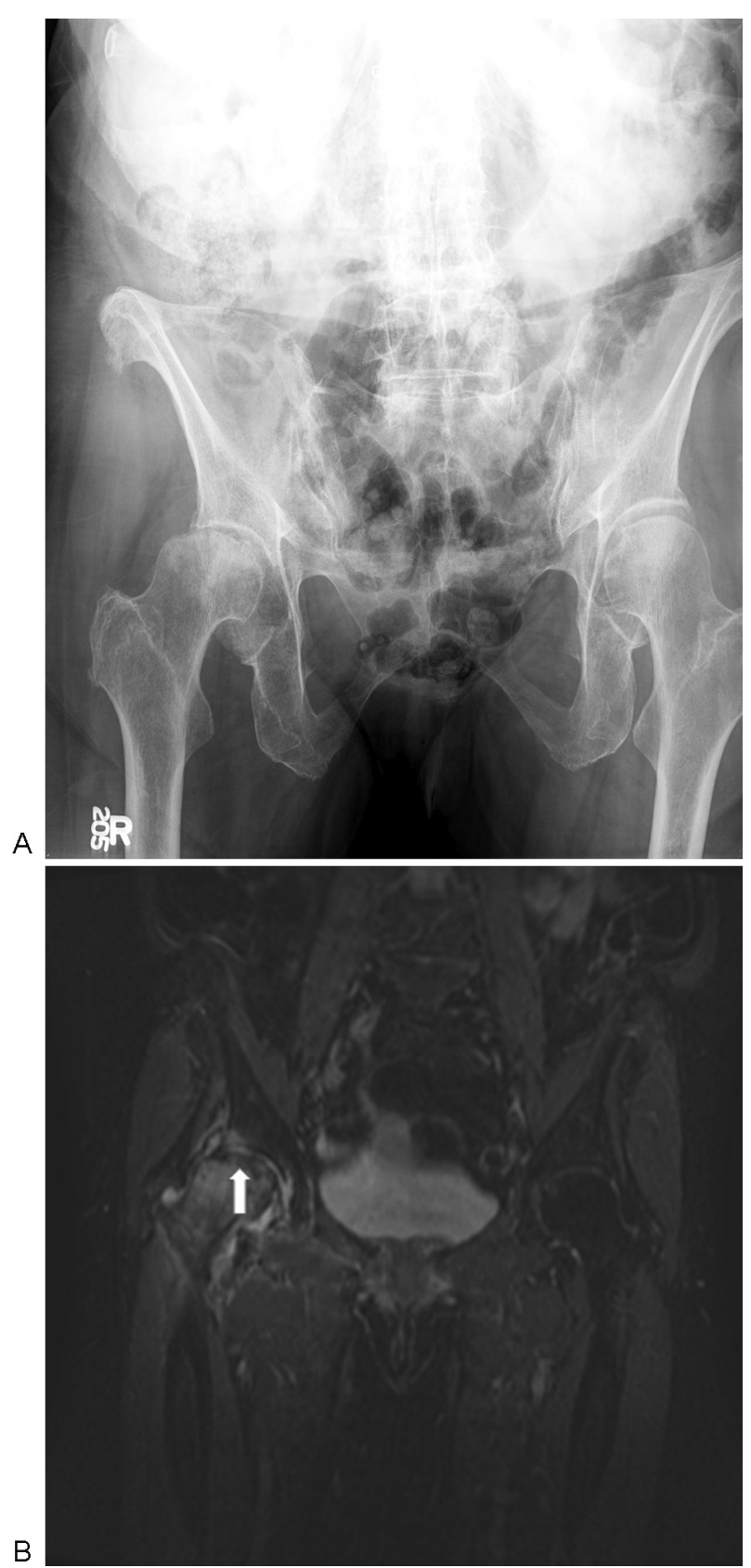

Fig. 1 (A) Radiograph obtained 6 weeks after the onset of severe right hip pain. (B) MRI obtained 4 days after radiograph. Notice appearance of subchondral fracture line (arrow) with bone edema distally.

removal or replacement that was not consistent with ON but consistent with a SIFH. In contrast to ON, SIFH individuals are often older and progress to bone collapse with bone removal that would require an intact blood supply. Bone absorption may progress to bone collapse and loss of femoral head congruity leading to joint replacement. ${ }^{3}$ SIFH in some individuals may progress as osteoarthritis (OA) with a slower course that may lead to cyst formation. ${ }^{4}$ In more severe cases patients will progress to $\mathrm{RDO}^{5}$ (-Figs. 1, 2).

Both ON and SIFH are often associated with a sudden onset of severe hip pain. ${ }^{6,7}$ Typically, an insufficiency fracture has a low-signal band on T1- and T2-weighted MRI in the subchondral bone close to the articular surface. This is associated with

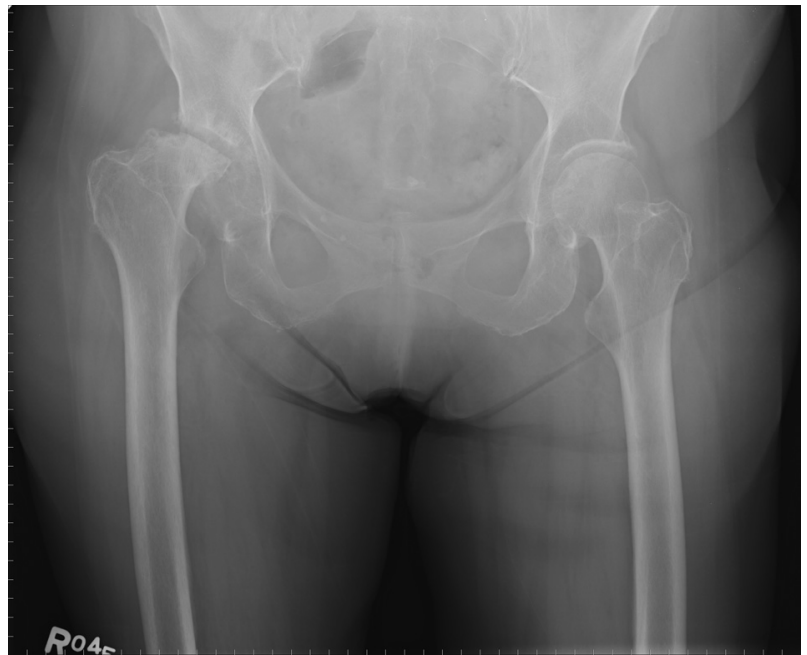

Fig. 2 Same hip seen in - Fig. 1, 6 months later. Not absorption of femoral head and portion of acetabulum.

bone marrow edema throughout the femoral head. Because the blood supply is initially intact, SIF has increased scintigraphic uptake in the femoral head. This is in contrast to decreased scintigraphic uptake in the affected portion of subchondral bone in $\mathrm{ON}^{3}$ In SIFH the low-intensity band on MRI appears to correspond to a fracture line with associated repair tissue. Histology in the region of high T2 signal intensity correlates with viable bone and marrow tissue with associated callus, edema, and vascular granulation tissue. ${ }^{8}$ SIFH was confirmed by histology as the primary process in $6.5 \%$ of 7,718 resected femoral heads. In all cases of SIFH, there was callus and granulation tissue along the fracture line. ${ }^{9}$ In a single-case study, hip replacement was performed 3 months after the onset of pain and progression to bone collapse within the region of the femoral head; typically on MRI for SIF, the intralesion section clearly demonstrated edematous changes and vascular rich granulation tissue. ${ }^{10}$

\section{Rapidly Destructive Osteoarthritis of the Hip}

In his 1957 thesis, Forestier first described a rapidly progressive destruction of both the femoral and acetabular aspects of the hip joint. Postel and Kerboull were the first to report a group of patients who developed a rapid loss of a portion of the femoral head. ${ }^{11}$ This is in contrast to transient osteoporosis wherein MRI evaluation revealed a generalized bone edema throughout the femoral head and neck but no line of demarcation. ${ }^{12}$ The histology of transient osteoporosis, also called bone marrow edema syndrome or transient demineralization, is distinct from that of SIFs. ${ }^{13}$ The key findings from core decompression sections located in the MRI edematous regions were bone trabeculae that had partly osteoblastcovered osteoid seams and irregular woven bone (microcallus), pointing to increased bone formation activity. The clinical course and radiographic findings for RDO are quite distinct. Overall, it appears that many cases of RDO follow SIFH. ${ }^{14}$ However, it is not clear whether SIFH precedes all cases of RDO. The relationship of RDO to osteoporosis is 
suspected given the age group of patients. However, there is a report of no marked differences in bone mineral density of the lumbar spine, ultra-distal radius, mid-radius, and calcaneus between patients with rapid destructive and primary OA. ${ }^{15}$ The relationship to abnormal hip architecture similar to that seen in OA has been noted for SIFH and RDO. ${ }^{16}$ There are other medical conditions that can lead to rapid destruction of the hip joint. Hence the distinction of RDO from these other disorders is important for the understanding of the RDO process. ${ }^{17}$ Examples include septic arthritis, neuropathic osteoarthropathy, ON, crystal induced arthropathy, and hyaluronic acid crystal induced arthropathy. More rare associations include amyloid arthritis, ochronosis, rheumatoid arthritis, psoriatic arthritis, and SAPHO (synovitis, acne, pustulosis, hyperostosis, and osteitis).

\section{Subchondral Insufficiency Fracture of the Knee}

The term spontaneous osteonecrosis of the knee (SONK) was first described by Ahlbäck et al in $1968 .{ }^{18}$ The term has been used to describe an apparent ON that mostly affects the medial portion of the femoral condyle and proximal tibia in more advanced OA. With the advent of the MRI, the term SONK was applied to a sequence of events that start with a sudden onset of acute, unilateral knee pain in individuals older than 50 years, and who are predominantly female. Most individuals have no recent trauma and do not have risk factors for ON. Many patients have an initially painful course followed by a resolution of symptoms over months. Unfortunately, progressive collapse can occur and continued severe symptoms may lead to surgical interventions. Evidence suggests that SONK should be linked with OA and insufficiency fractures. ${ }^{19}$ Others have also stated that SONK should be reclassified as an insufficiency fracture. ${ }^{20}$ In general terms, an insufficiency fracture is defined as a fracture that occurs in the bone due to the inability of the bone to support normal loads. ${ }^{21}$ An insufficiency fracture may also be defined as a fracture that occurs due to the bone of inadequate quality. Regardless of the definition, the term implies that an insufficiency fracture would occur only in individuals with poor bone quality or mass. The term SIFK is also applied to MRI of the proximal tibial plateaus and femoral condyles early in the disease process. The T1- or proton density-weighted image typically has a curvilinear area of decreased signal that parallels the articular margin of the subchondral bone (-Fig. 3). In contrast to secondary ON, all lesions have a direct connection to the articular subchondral bone. The average articular surface area can be $431 \pm 218 \mathrm{~mm}^{2}$ (range: $210-1,025)$ and $4.8 \pm 3.1 \mathrm{~cm}^{3}$ in volume. ${ }^{22}$ The pattern of the bone marrow edema seen in these lesions is different than the typical MRI of bone marrow lesions seen in $\mathrm{OA} .{ }^{23}$ The pattern described in the paper by Scher et al is distinct from the clear line of demarcation seen in most insufficiency fractures. Patients who present with an MRI appearance of SIFK are mostly past their fifth decade and usually do not report an injury sufficient to produce an intra-articular fracture. ${ }^{24}$ In summary, the link between SIFK and SONK has become

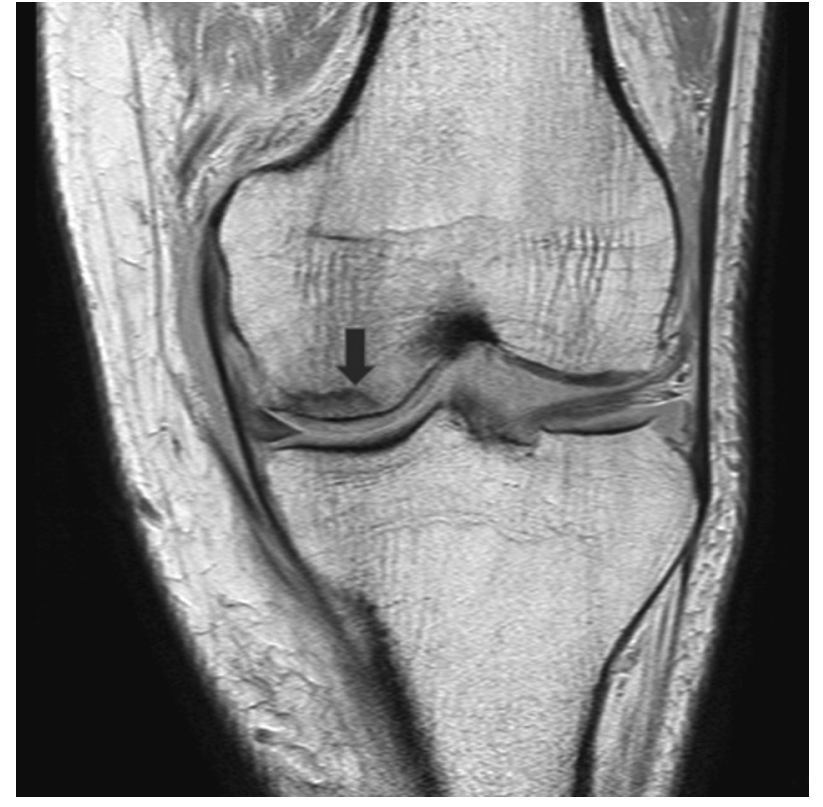

Fig. 3 Subchondral insufficiency fracture of distal femoral condyle.

clearer over the past 15 years. ${ }^{8,20,25}$ It has been shown that systemic bone mineral density and bone mineral density in the vicinity of new insufficiency fractures are reduced. ${ }^{26}$ This correlates with early OA wherein there is decreased periarticular bone density relative to systemic bone density. ${ }^{27}$ The overall implication of most studies is that insufficiency fractures around the knee are typically due to systemic insufficient bone and may lead to, or be associated with, SONK. However, a recent study has demonstrated that most patients with SIFK are not osteoporotic and, for age, have above normal bone density. ${ }^{16}$ The disconnection between low condylar bone density and a higher systemic bone density implies an important dynamic that may be related to early focal vascular signals.

\section{Bone Edema}

The term bone marrow edema has been applied to ill-defined increased signal intensities seen on short inversion time inversion-recovery images and on T2-weighted, fat-suppressed MRIs. ${ }^{23,28}$ These are considered to represent bone marrow edema because these changes can be reversible following distance running and a knee injury such as a ligament rupture. When areas of MRI of bone edema seen in $\mathrm{OA}$ are compared with areas of normal signals in $\mathrm{OA}$, histology shows significantly more bone marrow necrosis, bone marrow fibrosis, and abnormal trabeculae in areas of increased signal. ${ }^{28}$ Their definition of histologic bone marrow edema was areas of swollen fat cells interspersed with eosinophilic extracellular fluid. That finding is only a small percentage of MRI of areas of normal and increased signal. It is for this reason that some investigators prefer the term bone marrow edema-like signal or bone marrow lesion.

Very helpful is a recent review of the description of the histology seen on MRI of bone lesions. ${ }^{29}$ In the case of a bone 
contusion, there are subchondral microfractures, osteocyte necrosis and empty lacunae, hemorrhage, and edema. In the bone marrow lesions seen in $\mathrm{OA}$, there are changes indicative of bone remodeling. Later in the process, there may be increased thickness and mineral density of the subarticular trabeculae. Other changes include microcracks, microedema, and microbleeding within the subchondral region. When removed bone is replaced with subchondral bone cysts, these cysts have usually more intense MRI signal. In the case of SIFK, trabecular microcracks may contribute to subchondral alterations that may result in focal $\mathrm{ON}$ of the subchondral bone. Severe involvement of marrow cells and vessels may show evidence of necrosis and osteocytes become necrotic. These lesions can recover with new trabecular bone in the subarticular spongiosa. In some cases, there is insufficient support and recovery resulting in a nonviable bone sequestrum.

\section{Biochemical Change in SIFH, RDO, and SIFK}

There is a paucity of literature on any specific biochemical mechanisms that might be important in either SIFH, SIFK, or RDO. Synovial cells may be responsible for cytokine signals that would enhance rapid destruction of the bone. One study compared synoviocytes from typical OA to those from RDO. There was a greater secretion of matrix metalloproteinase (MMP)-2, MMP-3, and interleukin-1 (IL-1). ${ }^{30}$ Compared with $\mathrm{OA}$, there are much higher serum and plasma levels of MMP-2 and MMP-9 in RDO. ${ }^{31}$ In another study, synovial fluid was taken from patients with either ON, developmental dysplasia, and/or RDO. ${ }^{32}$ Bone alkaline phosphatase, MMP-3, and keratan sulfate were significantly higher in the ONF group than those in the RDA and DDH groups. Tartrate-resistant acid phosphatase $5 \mathrm{~b}$, a marker of osteoclasis, was highest in the RDO group. This correlates to elevated levels of serum crosslinking C-terminal telopeptide, a marker of bone resorption, which are higher in RDO than in OA. ${ }^{33}$ The levels of bone alkaline phosphatase in the ONFH group after the development of osteoarthritic changes were significantly lower than those observed in earlier stages. In comparisons between the samples obtained from each group with a terminal condition, the ONFH samples exhibited significantly higher MMP-3 and keratin sulfate levels, whereas the tartrate-resistant acid phosphatase 5b levels were highest in the RDO group. Given the potential impact of vascular ingrowth in SIFH, SIFK, and $\mathrm{RDO}$, it is surprising that so little literature is available relative to these conditions. However, there is information on the distinction between rheumatoid arthritis and OA. The differences in vascular changes seen in RA versus OA have been correlated to vascular endothelial growth factor (VEGF) ${ }^{34}$ In a review relative to the role of VEGF in OA and the potential for anti-VEGF drug therapies, it is noted that increased VEGF levels are associated with OA progression. ${ }^{35}$ VEGF appears to be involved in cartilage degeneration, osteophyte formation, subchondral bone cysts and sclerosis, synovitis, and pain. Inhibition of VEGF signaling reduces OA progression. Given that anti-VEGF therapy is being used for other conditions, the role for such therapy in OA, SIFH, SIFK, and RDO has yet to be determined.

\section{Vascularity}

\section{SIFH RDO}

Vascularity in SIFH and RDO is not extensively described relative to its importance in the process. A series of seven patients with SIFH that did not progress to RDO had histology compared with MRIs. ${ }^{8}$ The low-intensity band on T1-weighted MRI corresponded to a fracture line and its associated repair tissue. The proximal subchondral portion of the lesion had a homogeneous high signal intensity corresponding to viable bone and marrow tissue with associated callus, edema, and vascular granulation tissue.

In the case of RDO, it has been shown that synovial hyperplasia had a good correlation with osteocartilaginous debris and a poor correlation with amyloid infiltration. ${ }^{36}$ In these flattened femoral heads, there were large ischemic and necrotic areas with bone marrow atrophy and fibrosis. Of significance is the intense bone remodeling. Histology sections from a patient with rheumatoid arthritis who developed RDO revealed chronic synovitis with a foreign-body reaction in the soft tissues, and bone tissue examination revealed loss of articular cartilage, ON, fibrosis, and fatty degeneration. ${ }^{37}$ Of interest was that there was no mention of a hypervascular response.

\section{SIFK}

In a series of eight patients who had a joint replacement following SIFK, histology revealed a subchondral fracture with callus formation with the presence of woven bone. ${ }^{38}$ Four patients had "essentially intact articular cartilage overlying the lesion." The disruption of the trabecular architecture was indicative of subchondral bone fracture. The other patients had articular cartilage disruption with an isolated osteochondral defect and reparative tissue within the base of the defect. The two knees with ON were likely secondary to the fracture.

\section{Comparison with Other Conditions}

Several authors have recently emphasized the role of MRI in the diagnosis of subchondral fractures. There is increasing interest about this type of fracture. The question is whether the mechanisms of articular changes seen in the hip and knee relate to other well-known destructive articular conditions. This would include distal clavicular osteolysis, rapidly progressive OA of the hip, SONK, adult-type Freiberg's infraction, and a rare condition, rapid destruction of the humeral head. ${ }^{39}$ Albeit there are MRI similarities and the presence of osteoporosis in rapid destruction of the humeral head, it is uncertain that the mechanisms involved are the same.

\section{Treatments}

A common treatment for SIF is restricted weight bearing. Patients will often select mechanical support such as crutches or walkers. However, there is no known prospective study as to the effectiveness of weight bearing restriction. The benefits from bisphosphonates are not clear. Amino-bisphosphonates 
cause osteoclast apoptosis. These have been used is SIFK. In one study three or four intravenous infusions over 2 weeks of $120 \mathrm{mg}$ pamidronate followed by $70 \mathrm{mg}$ oral alendronate weekly for 4 to 6 months appeared to be effective in most patients. ${ }^{40}$ However, there were no controls. In a doubleblind, placebo-controlled study, compared with anti-inflammatory medications ibandronate had no over and above beneficial effect on clinical and radiologic outcome in patients with SONK. ${ }^{41}$ Iloprost is a prostacyclin. Prostacyclin is also called prostaglandin $\mathrm{I}_{2}$ or $\left(\mathrm{PGI}_{2}\right)$. It dilates blood vessels resulting in increased blood flow. A randomized study compared iloprost $(n=21$, group 1$)$ with tramadol $(n=20$, group 2). ${ }^{42}$ The treatment duration was 4 weeks. The analgesic effect of iloprost and tramadol was similar. Bone marrow edema regression on MRIs was more pronounced with iloprost treatment. ${ }^{42}$ Iloprost has been studied in secondary avascular necrosis and found to decrease pain in the earlier phases of the disease but not preventing long-term progression to joint replacement. ${ }^{43,44}$ Although not studied in SIF or RDO, VEGF has been studied in OA. Increased VEGF levels are associated with $\mathrm{OA}$ progression and are associated with cartilage degeneration, osteophyte formation, subchondral bone cysts and sclerosis, synovitis, and pain. ${ }^{45}$ Given the interest in the potential benefits of inhibition of VEGF and its receptors as an OA treatment, the study of VEGF in SIF and RDO is warranted. Gorham's disease is the absorption of the distal clavicle seen in younger individuals such as weight lifters. It has some of the characteristics of SIF and is associated with angiogenesis and massive lymph angiogenesis as well as the expression of vascular endothelial growth factor-A. In a single case the patient responded favorably, and the disease was stabilized following treatment with the $\beta$-blocking agent propranolol. Propranolol acts on VEGF-A. ${ }^{46}$

\section{Future Research as It May Change Therapy}

The common denominator for SINH and SIFK is a focal osteoporosis often independent of any systemic osteoporosis. The bone fragility may lead to focal microfracture wherein there is overall adequate support to prevent wide collapse and joint destruction. In the case of the hip, a slower OA-like response to SIFH may lead to joint replacement or no cause for intervention. On the other hand, the rapidly destructive process seen in RDO appears to be associated with a more vigorous osteoclasis that would require increased vascularity and the relative role of signals leading to increased osteoclasis. Because of the rarity of these conditions, little is known about the mechanical and cytokine triggers. One of the barriers to a more complete understanding is the inability to ethically use tissue samples in the earlier phases of the disorders. It is possible that receptor-tagged imaging may lead to a better understanding of the similarities and differences in these conditions.

Financial support

There was no funding or financial support for this review.

\section{Acknowledgment}

Dr. Lynne Jones reviewed the content during the writing of this paper.

\section{References}

1 Kuo A, Ezzet KA, Patil S, Colwell CW Jr. Total hip arthroplasty in rapidly destructive osteoarthritis of the hip: a case series. HSS J 2009;5(2):117-119

2 Batra S, Batra M, McMurtrie A, Sinha AK. Rapidly destructive osteoarthritis of the hip joint: a case series. J Orthop Surg 2008;3:3

3 Davies M, Cassar-Pullicino VN, Darby AJ. Subchondral insufficiency fractures of the femoral head. Eur Radiol 2004;14(2):201-207

4 Fukui K, Kaneuji A, Fukushima M, Matsumoto T. Imaging and histopathological evaluation of a cystlike formation in subchondral insufficiency fracture of the femoral head: a case report and literature review. Int J Surg Case Rep 2014;5(6):324-329

5 Ikemura S, Yamamoto T, Motomura G, Nakashima Y, Mawatari T, Iwamoto Y. MRI evaluation of collapsed femoral heads in patients 60 years old or older: differentiation of subchondral insufficiency fracture from osteonecrosis of the femoral head. AJR Am J Roentgenol 2010;195(1):W63-W68

6 Bullough PG, DiCarlo EF. Subchondral avascular necrosis: a common cause of arthritis. Ann Rheum Dis 1990;49(6):412-420

7 Yamamoto T. Subchondral insufficiency fractures of the femoral head. Clin Orthop Surg 2012;4(3):173-180

8 Yamamoto T, Schneider R, Bullough PG. Subchondral insufficiency fracture of the femoral head: histopathologic correlation with MRI. Skeletal Radiol 2001;30(5):247-254

9 Yamamoto T, Iwamoto Y, Schneider R, Bullough PG. Histopathological prevalence of subchondral insufficiency fracture of the femoral head. Ann Rheum Dis 2008;67(2):150-153

10 Zhao G, Yamamoto T, Ikemura S, et al. A histopathological evaluation of a concave-shaped low-intensity band on T1-weighted MR images in a subchondral insufficiency fracture of the femoral head. Skeletal Radiol 2010;39(2):185-188

11 Postel M, Kerboull M. Total prosthetic replacement in rapidly destructive arthrosis of the hip joint. Clin Orthop Relat Res 1970;72(72):138-144

12 Kim JW, Yoo JJ, Min BW, Hong SH, Kim HJ. Subchondral fracture of the femoral head in healthy adults. Clin Orthop Relat Res 2007; 464(464):196-204

13 Plenk H Jr, Hofmann S, Eschberger J, et al. Histomorphology and bone morphometry of the bone marrow edema syndrome of the hip. Clin Orthop Relat Res 1997;(334):73-84

14 Mavrogenis AF, Flevas DA, Panagopoulos GN, et al. Rapid destructive arthritis of the hip revisited. Eur J Orthop Surg Traumatol 2015;25(7):1115-1120

15 Okano K, Aoyagi K, Enomoto H, Osaki M, Chiba K, Yamaguchi K. Bone mineral density in patients with destructive arthrosis of the hip joint. J Bone Miner Metab 2014;32(3):312-316

16 Nelson FR, Craig J, Francois H, Azuh O, Oyetakin-White P, King B. Subchondral insufficiency fractures and spontaneous osteonecrosis of the knee may not be related to osteoporosis. Arch Osteoporos 2014;9:194

17 Porrino J, Carlson B, Kani KK, Mulcahy H, Wyatt A, Chew FS. Disappearing acts: the many causes of rapidly destructive arthritis. Curr Probl Diagn Radiol 2016:S0363-0188(15)30050-5

18 Ahlbäck S, Bauer GC, Bohne WH. Spontaneous osteonecrosis of the knee. Arthritis Rheum 1968;11(6):705-733

19 Yamamoto T, Bullough PG. Spontaneous osteonecrosis of the knee: the result of subchondral insufficiency fracture. J Bone Joint Surg Am 2000;82(6):858-866

20 Kidwai AS, Hemphill SD, Griffiths HJ. Radiologic case study. Spontaneous osteonecrosis of the knee reclassified as insufficiency fracture. Orthopedics 2005;28(3):236, 333-336 
21 Cabarrus MC, Ambekar A, Lu Y, Link TM. MRI and CT of insufficiency fractures of the pelvis and the proximal femur. AJR Am J Roentgenol 2008;191(4):995-1001

22 Aratake M, Yoshifumi T, Takahashi A, Takeuchi R, Inoue T, Saito T. Evaluation of lesion in a spontaneous osteonecrosis of the knee using 18F-fluoride positron emission tomography. Knee Surg Sports Traumatol Arthrosc 2009;17(1):53-59

23 Scher C, Craig J, Nelson F. Bone marrow edema in the knee in osteoarthrosis and association with total knee arthroplasty within a three-year follow-up. Skeletal Radiol 2008;37(7):609-617

24 Lafforgue P, Daumen-Legré V, Clairet D, Daver L, Acquaviva PC. Insufficiency fractures of the medial femoral condyle. Rev Rhum Engl Ed 1996;63(4):262-269

25 Narváez JA, Narváez J, De Lama E, Sánchez A. Spontaneous osteonecrosis of the knee associated with tibial plateau and femoral condyle insufficiency stress fracture. Eur Radiol 2003; 13(8):1843-1848

26 Zanetti M, Romero J, Dambacher MA, Hodler J. Osteonecrosis diagnosed on MR images of the knee. Relationship to reduced bone mineral density determined by high resolution peripheral quantitative CT. Acta Radiol 2003;44(5):525-531

27 Karvonen RL, Miller PR, Nelson DA, Granda JL, Fernández-Madrid F. Periarticular osteoporosis in osteoarthritis of the knee. J Rheumatol 1998;25(11):2187-2194

28 Zanetti M, Bruder E, Romero J, Hodler J. Bone marrow edema pattern in osteoarthritic knees: correlation between MR imaging and histologic findings. Radiology 2000;215(3):835-840

29 Kon E, Ronga M, Filardo G, et al. Bone marrow lesions and subchondral bone pathology of the knee. Knee Surg Sports Traumatol Arthrosc 2016;24(6):1797-1814

30 Komiya S, Inoue A, Sasaguri Y, Minamitani K, Morimatsu M. Rapidly destructive arthropathy of the hip. Studies on bone resorptive factors in joint fluid with a theory of pathogenesis. Clin Orthop Relat Res 1992;(284):273-282

31 Masuhara K, Nakai T, Yamaguchi K, Yamasaki S, Sasaguri Y. Significant increases in serum and plasma concentrations of matrix metalloproteinases 3 and 9 in patients with rapidly destructive osteoarthritis of the hip. Arthritis Rheum 2002;46(10):2625-2631

32 Yamaguchi R, Yamamoto T, Motomura G, et al. Bone and cartilage metabolism markers in synovial fluid of the hip joint with secondary osteoarthritis. Rheumatology (Oxford) 2014;53(12): 2191-2195

33 Berger CE, Kröner A, Stiegler H, Leitha T, Engel A. Elevated levels of serum type I collagen C-telopeptide in patients with rapidly destructive osteoarthritis of the hip. Int Orthop 2005;29(1):1-5
34 Zhao MS, Xia RH, Wang YH, Ding LX, Guan L, Huang F. [Characteristics of vascular morphology and vascular endothelial growth factor in patients with osteoarthritis and rheumatoid arthritis] [in Chinese]. Beijing Da Xue Xue Bao 2012;44(6):927-931

35 American College of Rheumatology Subcommittee on Osteoarthritis Guidelines. Recommendations for the medical management of osteoarthritis of the hip and knee: 2000 update. Arthritis Rheum 2000;43(9):1905-1915

36 Mitrovic DR, Riera H. Synovial, articular cartilage and bone changes in rapidly destructive arthropathy (osteoarthritis) of the hip. Rheumatol Int 1992;12(1):17-22

37 Yun HH, Song SY, Park SB, Lee JW. Rapidly destructive arthropathy of the hip joint in patients with rheumatoid arthritis. Orthopedics 2012;35(6):e958-e962

38 MacDessi SJ, Brophy RH, Bullough PG, Windsor RE, Sculco TP. Subchondral fracture following arthroscopic knee surgery. A series of eight cases. J Bone Joint Surg Am 2008;90(5): 1007-1012

39 Viana SL, Machado BB, Mendlovitz PS. MRI of subchondral fractures: a review. Skeletal Radiol 2014;43(11):1515-1527

40 Kraenzlin ME, Graf C, Meier C, Kraenzlin C, Friedrich NF. Possible beneficial effect of bisphosphonates in osteonecrosis of the knee. Knee Surg Sports Traumatol Arthrosc 2010;18(12):1638-1644

41 Meier C, Kraenzlin C, Friederich NF, et al. Effect of ibandronate on spontaneous osteonecrosis of the knee: a randomized, doubleblind, placebo-controlled trial. Osteoporos Int 2014;25(1): 359-366

42 Mayerhoefer ME, Kramer J, Breitenseher MJ, et al. Short-term outcome of painful bone marrow oedema of the knee following oral treatment with iloprost or tramadol: results of an exploratory phase II study of 41 patients. Rheumatology (Oxford) 2007;46(9): 1460-1465

43 Jäger M, Tillmann FP, Thornhill TS, et al. Rationale for prostaglandin $\mathrm{I} 2$ in bone marrow oedema-from theory to application. Arthritis Res Ther 2008;10(5):R120

$44 \mathrm{Claßen} \mathrm{T}$, Becker A, Landgraeber S, et al. Long-term clinical results after iloprost treatment for bone marrow edema and avascular necrosis. Orthop Rev (Pavia) 2016;8(1):6150

45 Hamilton JL, Nagao M, Levine BR, Chen D, Olsen BR, Im HJ. Targeting VEGF and its receptors for the treatment of osteoarthritis and associated pain. J Bone Miner Res 2016;31(5):911-924

46 Baud J, Lomri A, Graber D, Bikfalvi A. The therapeutic response in Gorham's syndrome to the beta-blocking agent propranolol is correlated to VEGF-A, but not to VEGF-C or FLT1 expression. BMC Res Notes 2015;8:333 